

\title{
STUDY ON CONSUMER TRUST IN B2C E-COMMERCE OF SPECIALTY PRODUCTS FOR NATIONALITY - TAKING THE EXAMPLE OF TIBETAN TEA
}

\author{
Zhan Ying, Wu Jian \\ Wuhan Business University, Department of Business Management, Hubei Province, Wuhan City, 430056
}

This is an open access article distributed under the Creative Commons Attribution License, which permits unrestricted use, distribution, and reproduction in any medium, provided the original work is properly cited.

\section{ARTICLE DETAILS}

\section{Article History:}

Received 26 June 2018 Accepted 2 July 2018 Available online 1 August 2018

\section{ABSTRACT}

Researching on consumer trust in B2C E-commerce of specialty products for nationality is beneficial to build a precision poverty alleviation system based on specialty products for nationality and relying on e-commerce platform to promote the development of national unity and precision poverty alleviation. On the basis of the academic monographs of domestic and foreign scholars, this paper takes the example of Tibetan tea and adopts AHP method underlying group decision-making to establish an evaluation index system from six dimensions: credibility, commitment, benefit, monitoring, ability and prediction, which are compared according to the significance, out of the Tibetan area and heading for the mainland, the significance of each dimension is expected to speed up the development of rural e-commerce in Tibet, and to provide references and guidance for the development of ecommerce precision poverty alleviation model projects.

\section{KEYWORDS}

B2C E-commerce, Specialty products for nationality, Trust, Tibetan tea, Group decision-making, AHP, E-commerce precision poverty alleviation.

\section{INTRODUCTION}

With the advancement of the project of basic promotion and the coordinated development of the region, the Tibetan area with rich national characteristics has steadily formed a way for the farmers to provide raw materials for enterprises, and to develop the national characteristic products for the consumers (i.e., the consumer B2C e-commerce mode) [1]. As the famous national characteristic products in the Midwest, the paper studies the Trust degree of the consumers to the Tibetan tea under the mode of $\mathrm{B} 2 \mathrm{C}$ electronic commerce, constructs the precision poverty alleviation system based on the electronic commerce platform, which can effectively realize the Tibetan tea coming out of Tibet and the mainland, for other national characteristics of the product of the development of electric dealers to provide accurate model of poverty alleviation Project effectiveness [2].

The heat of research on the trust degree of agricultural products by domestic scholars is mild. From Three aspects namely the certification system of ecological agricultural products, brand communication model, Community support Agriculture (CSA) to study the construction of the trust mechanism of ecological agricultural products; a group of scholars taking Taobao as the research object, they point out the defects of the trust evaluation of agricultural products, synthesize the research theories of the relevant scholars, and put forward suggestions and countermeasures for the agricultural Product credit evaluation system of Taobao; another group of scholars consider the subjective and uncertain characteristics of the trust of agricultural products in the course of electronic commerce, and put forward a model of agricultural trust evaluation based on "cloud model and Bayesian network model" [3-5]. Through the search of China's knowledge network found that the domestic scholars on the topic of trust has been endless, in recent years, more than 6000 papers published volume. This paper studies the topic of research and trust of domestic agricultural products, absorbs Zhou Guilin's academic monographs, adopts AHP method based on group decision, constructs the evaluation index system of Tibetan tea from 6 dimensions of trust, promise, benefit, monitor, ability and forecast, and quantifies the importance of each dimension, thus, apply medicine according to indications, grasp the key and put forward the reasonable suggestion.

\section{RESEARCH METHODS}

\subsection{Document matching method}

Using the Wuhan Business University Library, China Knowledge Network, Google Academic, Wanfang and other resource platforms, through the information retrieval matching and the contents of the study related to the literature, intensive reading of Zhou Guilin's academic monographs--"Based on community identity of the trust model of E-commerce," reserve a certain domestic and foreign research on the theory of trust.

\subsection{Group decision Law}

For some complex and changeable problems, it often involves the multiplicity of goals, the dynamics of time and the uncertainty of State, simply by a Human ability is far from being able to make scientific and rational decision, it needs to integrate group intelligence to participate in decision analysis, and to bridge the difference of individual decision, then to make the decision more positive reality-oriented.

\subsection{AHP method}

Compare the indicators in the evaluation system, by using the 9-point ratio to schedule the relative merits and demerits of each evaluation index, the judgment matrix is further calculated, then the consistency test is carried out, and the weight vector is calculated according to the formula of the combination weight vector, which can quantify the important degree of 6 dimensions, such as credibility, promise, benefit, monitoring, ability and forecast.

\section{RESEARCH ON CONSUMERS ' TRUST TO TIBETAN TEA UNDER THE MODE OF B2C BUSINESS ELECTRONIC COMMERCE}

\subsection{Construction of Evaluation Index system}

Based on the research of domestic agricultural products and the topic of trust, absorbing Zhou Guilin's academic Monographs, combining Wuhan Business School with Sino-Tibetan Cultural Exchange Center, Wuhan Tanggula Culture Exchange Co., Ltd. Long-term exchange of information 
obtained and transformation of the results, from 6 dimensions of trust, commitment, benefit, monitoring, ability and forecast, the paper constructs the evaluation Index system of consumers' confidence in the tea-possession in the consumer e-commerce mode.

\subsection{Weight calculation of judgment matrix}

Taking advantage of the Wuhan Business University and the Sino-Tibetan Cultural Exchange Center to build the Yangtze River National characteristic product electric Business enterprise Cooperation opportunity, consulted 2 Chinese and Tibetan Cultural Exchange Center experts, 2 Wuhan Tanggula Han Tibetan Cultural Exchange Co., Ltd. deep, 1 Wuhan Business University E-commerce Professional leaders, in the form of questionnaires to grade, A total of 5 judgment matrices are constructed. Calculate the weight vectors of each matrix, according to 6 index weights, the weight relative size is sorted, the sort order is $" 1,2,3,4,5,6 "$ and the weight is increased successively.

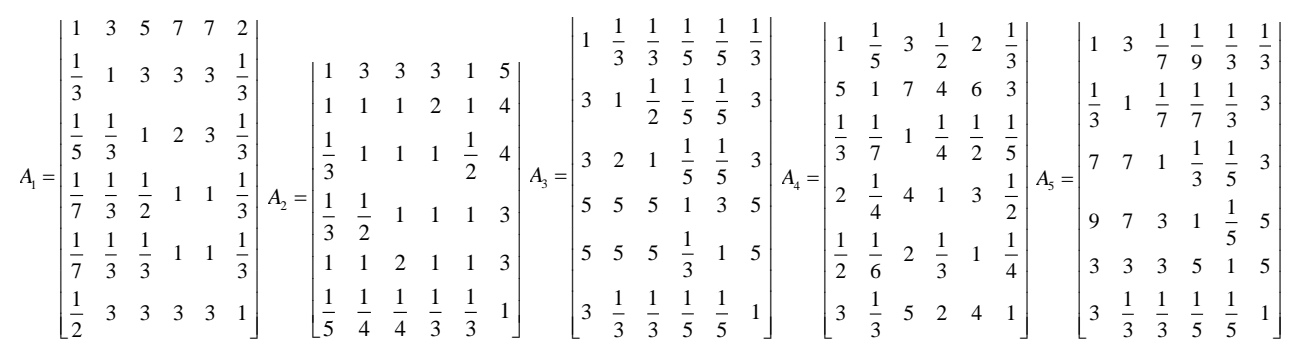

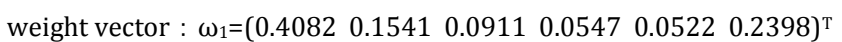

Index Sort: 643215

weight vector : $\omega_{2}=\left(\begin{array}{llllll}0.2770 & 0.2077 & 0.1373 & 0.1309 & 0.1980 & 0.0491\end{array}\right)^{\mathrm{T}}$

Index Sort : 6553241

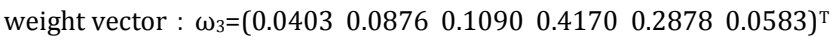

Index Sort : 134652

weight vector : $\omega_{4}=\left(\begin{array}{llllll}0.0490 & 0.1523 & 0.4295 & 0.0329 & 0.0999 & 0.2364\end{array}\right)^{\mathrm{T}}$

Index Sort : 246135

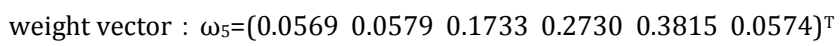

Index Sort : 134562

\subsection{Calculation of Kendall Harmony coefficient}

There is no same grade rating for the same reviewers in this paper. It is found that the Kendall Harmony coefficient $W$ formula is

$$
\begin{aligned}
& W=\frac{S S_{R}}{k^{2}\left(n^{3}-n\right)} \\
& S S_{R}=\sum\left(R_{i}-\sum R_{i} / n\right)^{2} \quad(i=1,2,3, \ldots, n)
\end{aligned}
$$

In the formula, $\mathrm{k}$ represents the total number of reviewers, $\mathrm{n}$ indicates the total number of evaluation indicators, and Ri represents the ranking of weights for the i evaluation index.

\begin{tabular}{|c|c|c|c|c|c|c|c|}
\hline \multirow{2}{*}{$\begin{array}{l}\text { Index } \\
n=6\end{array}$} & \multicolumn{5}{|c|}{ Reviewers $k=5$} & \multirow{2}{*}{$R$} & \multirow{2}{*}{$R^{2}$} \\
\hline & $\overline{A_{1}}$ & $\mathrm{~A}_{2}$ & $\mathrm{~A}_{3}$ & $\mathrm{~A}_{4}$ & $\mathrm{~A}_{5}$ & & \\
\hline credibility & 6 & 6 & 1 & 2 & 1 & 16 & 256 \\
\hline commitment & 4 & 5 & 3 & 4 & 3 & 19 & 361 \\
\hline benefit & 3 & 3 & 4 & 6 & 4 & 20 & 400 \\
\hline monitoring & 2 & 2 & 6 & 1 & 5 & 16 & 256 \\
\hline ability & 1 & 4 & 5 & 3 & 6 & 19 & 361 \\
\hline prediction & 5 & 1 & 2 & 5 & 2 & 15 & 225 \\
\hline Sum & & & & & & 105 & 1859 \\
\hline
\end{tabular}

Table 1: Kendall Harmony coefficient calculation table

The data in the table can be calculated in the formula (1): $w=0.04914$.

\subsection{The test of the significance of Kendall Harmony coefficient}

When the Kendall Harmony coefficient is tested, it must be changed to test, whether or not the same grade, the basic steps are as follows:

\section{(1) Making assumptions}

$$
H_{0}: \rho=0 \quad H_{1}: \rho \neq 0
$$

(2) Select a validation statistic and calculate its value

$$
\chi 2=\mathrm{k}(\mathrm{n}-1) \mathrm{W}=1.2285
$$

(3) Given the level of significance, determine the critical value

According to the degree of freedom $\mathrm{df}=5$, the table of $\chi 2$ distribution critical value shows that when the significant level $a=0.05, \chi 2$ (0.05) $=11.0705 ;$ When the significant level $a=0.01, \chi 2 \quad(0.01)=15.0863$.

(4) Statistical decision
Since $\chi 2=1.2285<\chi 2(0.05)<\chi 2(0.01)=15.0863$, the existing data accept $\mathrm{H} 0$ and reject $\mathrm{H} 1$, that is, 5 experts are significantly inconsistent in their assessment, the result of the weighting result is very different, so we need to make further calculation and make full use of the existing data.

\subsection{Weighting the weights of the weighted experts}

The paper found that, from 2005-2018 period, a total of 17 scholars have studied the determination of expert weights in group decision making, in the intensive reading of their research results, deductive reasoning process, fusion of the advantages of various methods, summed up the mathematical model of each expert weight calculation is as follows [6-10]:

The weight mean of the first indicator is:

$$
\overline{\omega_{l}}=\frac{1}{k} \sum_{1}^{k} \omega_{i j} \quad(i=1,2,3, \ldots, n j=1,2,3, \ldots, k)
$$

The mean vectors of the weights of $n$ indices are: $\bar{\omega}=\left\{\overline{\omega_{1}}, \overline{\omega_{2}}, \ldots, \overline{\omega_{n}}\right\}$

The absolute value of the difference between the weights and the mean vectors of the first J expert is: 
$\left|\omega_{j}-\bar{\omega}\right|=\sqrt{\left(\omega_{1 j}-\overline{\omega_{1}}\right) 2+\left(\omega_{2 j}-\overline{\omega_{2}}\right)^{2}+\cdots\left(\omega_{i j}-\overline{\omega_{1}}\right) 2} \quad(i=1,2,3, \ldots, n$ $j=1,2,3, \ldots, k)(3)$

The absolute value of the difference in the weights of the indicators for any two experts (expert $G$ and expert $h$ ) is:

$\left|\omega_{g} \quad-\omega_{\mathrm{h}}\right|=\sqrt{\left(\omega_{1 g}-\omega_{1 h}\right) 2+\left(\omega_{2 g}-\omega_{2 h}\right) 2+\cdots\left(\omega_{i g}-\omega_{i h}\right) 2}$

$i=1,2,3, \ldots, n)$

Take $\left.A_{j}=a_{j} \sum_{1}^{k}|\omega g-\omega h|+b_{j}\left|\omega_{j}-\bar{\omega}\right|, \quad j=1,2,3, \ldots, k\right)$, where the values of $a j+b j=1, \quad a j \geq 0, \quad b \geq 0$,

$\mathrm{Aj}$ and $\mathrm{bj}$ can be determined by the relevant personnel depending on the specific problem. In this paper, take $\mathrm{a}_{\mathrm{i}}=\mathrm{b}_{\mathrm{j}}=\frac{1}{2}$, then the weight of the first $\mathrm{J}$ weighting experts is: $\mathrm{T}: \mathrm{T}_{\mathrm{j}}=\frac{1}{A_{j}}$. Substituting data into formulas (2), (3), (4) is available:

$$
\begin{aligned}
& \bar{\omega}=\left\{\begin{array}{llllll}
0.16628 & 0.13192 & 0.18804 & 0.18170 & 0.20388 & 0.12820
\end{array}\right\} \\
& \left|\omega_{1}-\bar{\omega}\right|=0.3464 \quad\left|\omega_{2}-\bar{\omega}\right|=0.1716 \quad\left|\omega_{3}-\bar{\omega}\right|=0.6385 \\
& \left|\omega_{4}-\bar{\omega}\right|=0.3422 \quad\left|\omega_{5}-\bar{\omega}\right|=0.2501 \\
& \sum_{1}^{k}|\omega 1-\omega \mathrm{h}|=2.2394 \quad \sum_{1}^{k}|\omega 2-\omega \mathrm{h}|=1.4906 \quad \sum_{1}^{k} \mid \omega 3- \\
& \omega \mathrm{h} \mid=1.7550 \\
& \sum_{1}^{k}|\omega 4-\omega \mathrm{h}|=1.9982 \quad \sum_{1}^{k}|\omega 5-\omega \mathrm{h}|=1.6087
\end{aligned}
$$

Thus, to obtain:

$$
\mathrm{T}_{1}=0.7735 \quad \mathrm{~T}_{2}=1.2032 \quad \mathrm{~T}_{3}=0.8356 \quad \mathrm{~T}_{4}=0.8546 \quad \mathrm{~T}_{5}=1.0760
$$


vector of the 5-bit weighting experts: $\lambda^{*}=\left(\begin{array}{llll}0.1631 & 0.2537 & 0.1762 & 0.1802\end{array}\right.$ 0.2269).

\subsection{Calculate the composite weight}

According to the weighted sum method, the integrated weighting information is calculated, and the combined weights are fully considering the opinions of 5 empowering experts and make them interactive, more scientific and effective quantification of credibility, commitment, interest, monitoring, capacity, forecasting relative to the consumer E-commerce model of the trust of tea in the research of the importance of the degree [11].

Comprehensive weight

$$
\omega=\lambda^{*} \times\left\lfloor\begin{array}{c}
\omega_{1} \\
\omega_{2} \\
\omega_{3} \\
\omega_{4} \\
\omega_{5}
\end{array}\right\rfloor
$$

The $\omega 1, \omega 2, \omega 3, \omega 4, \omega 5, \lambda^{*}$ formula (5) can be calculated by:

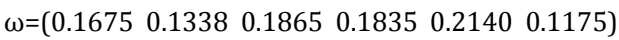

That is, the importance of the 6 dimensions from large to small order for capacity, interests, monitoring, credibility, commitment, prediction [12].

\section{CONCLUSIONS}

National characteristics of products industry as a very national cultural characteristics of the industry, with the business model of the merchant $\mathrm{E}$ commerce, the development momentum is getting better, especially the Yangtze River national characteristics of products, the establishment of the base and the strategic financial support. As one of the national characteristics of the Tibetan tea is bound to be in the e-commerce environment ushered in a new era of development. This paper studies consumer's trust in the possession of tea from 6 dimensions, such as credibility, promise, benefit, monitoring, ability and forecast. Among them, Credible representation the credibility of consumers and network sellers under virtual communities, the commitment indicates whether the network vendors can fulfill their commitments to consumers, benefits indicate whether the network vendors put the consumer interests first monitoring the behavior of network vendors is easy to monitor, ability to indicate whether network vendors have the ability to perform, predicting the predictability of network retailer behavior. The AHP method based on group decision is used to quantify: 6 dimensions of importance ranging from large to small order for ability, benefits, monitoring, trust, commitment, prediction, on this basis, the author puts forward 4 points to improve consumers ' trust to Tibetan tea in the mode of business ebusiness: The Government and enterprises work together to optimize the land integration and resource allocation in the Tibetan tea planting area. increasing farmers ' planting yield to ensure abundant reserves of Tibetan tea; With Jingdong, Alibaba, Suning and other warehousing logistics companies signed an annual cooperation agreement to improve the performance of logistics delivery efficiency; Broaden the channel of talent introduction, strengthen the field of cooperation between school and Enterprise, enhance the ability of online performance. Building Enterprise Culture, the interests of consumers always put in the first place, adhere to the original eco-national characteristics of the road, online price. Strictly check the price supervision and trademark authorization, to avoid the same brand price level of Tibetan tea is not uniform, to create a good trading order on the Tibetan tea line, whaley consumers. Perfecting the Tibetan credit system under the mode of B2C e-business, effectively exerting the relation degree between the Tibetan tea and his national characteristic products, creating the fine items and promoting the trust of the consumers in the virtual community to the Tibetan tea. In addition, attach importance to the development of "online electric business platform plus offline entity shop", into the new retail thinking, to provide consumers with a zero distance, high reality, good word-of-mouth experience. Pay attention to the interaction of consumer community platform, improve the trust degree and satisfaction of consumers, cultivate loyal customers, and breed "fan economy"; The algorithm of applying large data to the consumer as the center, accurately grasp the immediate needs of consumers, fixed-point precision implementation of marketing strategy.

\section{ACKNOWLEDGEMENTS}

Project fund: market analysis and product positioning analysis and promotion of Tibet Shannan ethnic products e-commerce platform (item No.: 2017KY004)

\section{REFERENCES}

[1] Zizara. 2018. Government work report. Tibet Daily (Han).

[2] Zhuang, H.J., Zeng, F.S. 2017. Wuhan Business School and the SinoTibetan Cultural Exchange Center to build the Yangtze River National characteristics Products Electric base.

[3]2018.

http://www.hbmzw.gov.cn/mcgz/mcsy/mcjj/39167.htm.

[4] Liao, H.F. 2018. On the construction of the trust mechanism of ecological agricultural products. China Prices, (1), 85-87.

[5] Lu, M.M., Zhang, T.T., Yan, Y.J. 2016. Analysis on the trust evaluation system of agricultural products based on Taobao. Science and Technology Horizon, (8), 273-282.

[6] Yang, X., Meng, Q.B., Sun, P., Wei, J.Y. 2014. Research on the trust evaluation model of agricultural products under electronic commerce Environment. Business Age, (34), 87-88.

[7] Zhou, G.L. 2012. E-commerce Trust model based on community identity. Harbin, Heilongjiang University Press.

[8] Liu, H., He, G.J. 2003. The analysis of result vectors in fuzzy comprehensive evaluation - Discuss with New Yan and Wang Zhenfu comrades. Statistics and decision making, (2), 63.

[9] Yan, R.X., Liu, J.L., Yao, B.X. 2007. Method and application of expert weight determination in group decision making. Statistics and Strategies, (23), 84-86.

[9] Zhu, F.F., Qu, S.Y., Zhang, F.W. 2008. A new method of expert weight determination in group decision making. Statistics and Decision Making, (7), 9-11. 
[11] Jiang, W.N. 2013. The idea and method of determining expert weights in group decision making. Statistics and Decision Making, (2), 2428.
[12] in committees. European Journal of Operational Research. 